\title{
RHINOPLASTY OF POSTTRAUMATIC NOSE INJURIES
}

\author{
E.Sh. Boliev \\ Department Of Otolaryngology And Stomatology \\ Tashkent Medical Academy, Uzbekistan \\ Sh.A. Makhamadaminova \\ Department Of Otolaryngology And Stomatology \\ Tashkent Medical Academy, Uzbekistan
}

Abstract: The aesthetic and functional significance of the external nose gives particular relevance to the treatment of its injuries. The social importance of the problem is caused by the widespread prevalence of nasoseptal injuries, the frequency of development of serious post-traumatic disorders of the appearance and normal physiology of patients, and unsatisfactory results of treatment of this pathology. The pyramid of the nose is the most vulnerable and fragile part of the facial skull, therefore, fractures of the nasal bones constitute a significant proportion of all injuries of the musculoskeletal system and occupy the third place among the total number of fractures of the human skeleton, are the most common among emergency conditions of ENT organs. In the general population, patients with this pathology account for up to $0.021 \%$.

Keywords: fracture of the bones of the nose, rhinoplasty, reconstructive inosurgery.

Objective of the study: improving the functional and aesthetic results of treatment of patients with fresh fractures of the nasal skeleton by improving organizational, diagnostic and therapeutic measures in accordance with the principles of modern reconstructive rhinosurgery.

Material and Methods: All 40 patients with nasal fractures underwent clinical and functional, laboratory and instrumental studies, rhinoplasty was performed in the otorhinolaryngology department of the TMA multidisciplinary clinic.

The results of an epidemiological study confirm a significant prevalence of nasal fractures in the structure of general ENT pathology (10.1\% in 2003, 9.6\% in 2004 and 6.3\% in 2005) and in the population of an industrial city of Russia (0.057\%, $0.061 \%$ and $0.059 \%$ ), which significantly exceeds the information of previous years and foreign data. Clinical 
examination data, as well as the degree of functional and aesthetic disorders, play a decisive role in determining the tactics and predicting the outcome of treatment of patients with nasoseptal injury (95\%). The results of traditional radiography, as well as sonography and computed tomography of the nasal bones, have practically no effect on tactics (preservation of the pretest treatment model in $100 \%$ of cases) and the effectiveness of treatment ( $p>0.05$ ) of traumatized patients in relation to leading clinical outcomes.

For objective detection, assessment and documentation of changes in appearance and nasal breathing in case of nasal injuries, as well as to control the quality of treatment, anterior active rhinomanometry and the author's technique of virtual computer rhinometry have shown high efficiency. Factors potentially contributing to unsatisfactory outcomes of traditional treatment of nasal fractures are too early (before 5 days) and later (after 10 days) intervention, as well as the curvature of the nasal septum and saddle deformity of the nasal dorsum prior to the injury. The presence of fresh lesions of the nasal septum does not impair the functional and aesthetic result of $K N$ reduction $(p>0.05)$. The proposed technique of conduction anesthesia of reposition of the nasal bones with carpulated articaine provides more effective analgesia $(p<0.05)$ and better results of treatment of patients ( $\% 2=5.19 ; v=1 ; p=0.023$ ) with fractures of the nasal bones in comparison with traditional methods. The results of a controlled prospective study do not allow us to consider intraoperative sonography as a method that improves the aesthetic $(\% 2=0.028 ; p=0.876)$ and functional $(x 2=0.001 ; p=0.975)$ results of ZRKN. Along with low utility, the technique requires additional equipment and increases the workload on staff. In case of fractures of the nasal bones, combined with severe deformity, hematomas and abscesses of the nasal septum, the use of septoplasty in combination with the reconstruction of the pyramid of the nose is more effective in comparison with the traditional treatment tactics, both in relation to nasal breathing $(x 2=15.3 ; \mathrm{V}=\mathrm{l} ; \mathrm{p}=0.00)$ and the appearance $(\wedge 2=6.3 ; V=I ; p=0.012)$ of the patient. The methods of minimally invasive open reconstruction of the injured nasal skeleton make it possible to achieve full functional and aesthetic rehabilitation of patients with the ineffectiveness of conventional treatment, the beginning consolidation and pathological fixation of the fragments ( $91 \%)$. 
With the joint elimination of post-traumatic nose deformities and cosmetic and functional disorders preceding the injury, the number of satisfactory results was $84 \%$ for the shape of the nose and 90\% for nasal breathing, which generally corresponds to the effectiveness of planned rhinoseptoplasty and justifies the application of the principle of one-stage aesthetic and functional rehabilitation in practice. The optimal period for reposition of the nasal bones is a period from 5 to 10 days after injury, when the swelling of the soft tissues of the injured nose is already decreasing, and the consolidation of bone fragments has not yet occurred. Earlier interventions may increase the likelihood of a poor outcome. When determining the treatment tactics in relation to patients with nasal injuries, as well as to control the quality of treatment, it is advisable to use methods of objective assessment and documentation of functional and aesthetic disorders - anterior active rhinomanometry and computed rhinometry. When a fracture of the nasal bones is combined with severe septal deformity, manifesting clinically, regardless of the age of its occurrence, a one-stage reduction of the nasal bones and septoplasty is indicated. The presence of an abscess and hematoma of the nasal septum is also an indication for the reconstruction of the septal skeleton in the acute period of trauma. In the absence of pronounced functional disorders, abscesses and hematomas of the nasal septum, all patients with fractures of the nasal bones are shown as the first stage of surgical treatment to perform closed reduction of the nasal bones in the operating room and with the possibility of expanding the scope of the intervention.

To improve the results of treatment of patients with nasoseptal injuries and for adequate anesthesia of the procedure for reposition of the nasal bones, this intervention should be carried out using carpool conduction anesthesia with articaine-containing drugs. With severe incorrect fixation of bone fragments and ineffectiveness of the primary closed reduction of the nasal skeleton, the method of choice is to carry out reconstructive rhinoseptoplasty, the volume of which depends on the characteristics of the traumatic process and the type of deformity. The decision to perform such an operation can be made intraoperatively or after control removal of fixing structures. Fractures of the nasal bones, accompanied by saddle deformity and destruction of the nasal septum, should be classified as clinical situations with an unfavorable functional and aesthetic prognosis. Surgical tactics should include open reconstruction of the nasal skeleton and 
extracorporeal septoplasty using absorbable synthetic material, autocartilage, or titanium miniplates. With a combination of a fresh fracture of the bones of the nose and a previous deformation of the external nose, it is possible to reconstruct the structures of the nose, except for situations when the elimination of the cosmetic defect is impeded by the mobility of the injured pyramid of the nose or the masking defect, edema of soft tissues.

\section{References:}

1. Botirov, A. J., et al. "Clinical and morphological results of xenografts to use in myringoplasty." The International Tinnitus Journal 24.1 (2020): 1-6.

2. Djuraev, Jamolbek Abdukaxorovich, et al. "Results of Allergological and Immunological Research in Patients with Polipoid Rhinosinusitis." Asian Journal of Immunology (2020): 34-40.

3. Djuraev, Jamolbek Abdukhahharovich, Ulugbek Saidakramovich Khasanov, and Ulugbek Nuridinovich Vokhidov. "The prevalence of chronic inflammatory diseases of the nose and paranasal sinuses in patients with myocarditis." European Science Review 5-6 (2018): 147-149.

4. Normurodov, Bakhtoyor K., et al. "Prevalence and structure of purulent inflammatory diseases of the maxillofacial area." Central Asian Journal of Medicine 2020.1 (2020): 116130.

5. Jumanov, Daulet Azadbek Ugli, et al. "International Journal of Biological and Pharmaceutical Sciences Archive." International Journal of Biological and Pharmaceutical Sciences Archive 1.1 (2021): 011-015.

6. Djuraev, J. A., et al. "Results of an immunogistochemical study in patients with polipoid rhinosinusitis." European Journal of Molecular \& Clinical Medicine 7.2 (2020): 2526-2541.

7. Khasanov, U. S., and J. A. Djuraev. "Morphological characteristics of chronic polypous rhinosinusitis." cutting edge-science (2020): 30.

8. Shaumarov, A. Z., et al. "Role of Hemostatic Agents in Simultaneous Surgical Interventions in the Nasal Cavity."

9. Kurbonov, Yokubjon Khamdamovich, Shukhrat Abdujalilovich Boymuradov, and Jamolbek Abdukakharovich Djuraev. "Purulent-Necrotic Diseases Of The Face: Aspects Of Diagnostics And Treatment." The American Journal of Medical Sciences and Pharmaceutical Research 3.01 (2021): 24-30. 
10. Narmurotov, Bakhtiyar Karshievich, Shukhrat Abdujalilovich Boymuradov, and Jamolbek Abdukakhkhorovich Djuraev. "Comparative Characteristics Of Rheological Properties Of Blood In Combined Face Injuries Before And After Treatment." The American Journal of Medical Sciences and Pharmaceutical Research 3.01 (2021): 67-75.

11. VOHIDOV, Ulugbek Nuridinovich, et al. "Current issues of the treatment of chronic polypous rhinosinusitis." Journal of Biomedicine and Practice 2.5 (2020).

12. Kurbonov, Yokubjon Khamdamovich, Shukhrat Abdujalilovich Boymuradov, and Jamolbek Abdukakharovich Djuraev. "Overview Of Comprehensive Treatment Of Acute Purulent-Inflammatory Diseases Of The Face And Neck." The American Journal of Medical Sciences and Pharmaceutical Research 3.01 (2021): 15-23.

13. Nigmatov, Iftikhor Obidjonovich, et al. "Post-Traumatic Defects And Face Deformations: Features Of Diagnostics And Treatment." The American Journal of Medical Sciences and Pharmaceutical Research 3.01 (2021): 55-66.

14. Djuraev, J. A. "Improvement of comprehensive treatment vasomotor rhinitis."

15. Khasanov, U. S., U. N. Vokhidov, and J. A. Djuraev. "State of the nasal cavity in chronic inflammatory diseases of the nose and paranasal sinuses in patients with myocarditis." European science,(9 (41)).-2018 (2018).

16. Vohidov, U. N., and J. A. Djuraev. "ugli, Makhsitaliev, MI, \& Khamidjanov, s." (2020).

17. UN, Vokhidov, et al. "The local immunity in the tissues of various forms of nasal polyps." ALLERGY. Vol. 71. 111 RIVER ST, HOBOKEN 07030-5774, NJ USA: WILEYBLACKWELL, 2016.

18. Vohidov, U. N. "Djuraev JA ugli, Makhsitaliev, MI, \& Khamidjanov, s. O.(2020). Current issues of the treatment of chronic polypous rhinosinusitis." Journal of Biomedicine and Practice 2.5.

19. Makhsitaliev, Mukhammadbobur, et al. "The Functional State Of The Mucous Membrane Of The Nasal Cavity And Paranasal Sinuses After Radical And Minimally Invasive Surgical Interventions." The American Journal of Medical Sciences and Pharmaceutical Research 3.01 (2021): 31-40. 